\title{
Interfacial Electro-Epoxidation in Electrospray Ionization Mass Spectrometry for Simultaneous Analysis of Negatively and Positively Charged Unsaturated Glycerophospholipids
}

\author{
Shuli Tang, ${ }^{\text {ta }}$ Licheng Fan, ${ }^{\text {ta }}$ Heyong Cheng, ${ }^{\text {a, b }}$ Xin Yan ${ }^{* a}$ \\ [a] Department of Chemistry, Texas A\&M University, 580 Ross St., College Station, TX 77845 \\ [b] College of Material Chemistry and Chemical Engineering, Hangzhou Normal University, Hangzhou, 311121, China \\ +These authors contributed equally to this work
}

\begin{abstract}
In this study, we developed an AC-induced interfacial electro-epoxidation reaction for localization of double-bonds in negatively and positively charged forms of lipids simultaneously. An AC voltage plays multiple roles in this method, including initiation of the electrochemical epoxidation of double-bonds in both charge states of lipids, and protonation and deprotonation of lipid for detection in both ion modes. The advantages of simultaneous detection of negatively changed and positively charged unsaturated lipids, voltage-controlled electrochemical derivatization, and the low sample consumption allow its wide applications in a broad range of lipid-related research.
\end{abstract}

\section{Introduction}

Lipids are a diverse and ubiquitous group of biomolecules including fatty acids, triacylglycerols, glycerophospholipids, phenols, sphingolipids, sterols, eicosanoids. ${ }^{1}$ They are the primary components of cell membranes and function crucially in cellular physiology such as energy storage, signal transduction, and metabolite transpot. ${ }^{2,3}$ Many studies have shown that altered lipid compositions are highly related with pathogenesis. ${ }^{4,5}$ For instance, human diseases such as obesity, ${ }^{6}$ diabetes, ${ }^{7}$ cardiovascular diseases, ${ }^{8}$ cancers, ${ }^{9}$ neurodegenerative disorders, ${ }^{10}$ and autoimmune disease ${ }^{11}$ show aberrant lipid metabolism. Hence, it is highly desirable to fully characterize the compositions and structures of lipids for the sake of diagnosing diseases and identifying disease mechanisms. ${ }^{12-15}$

Elucidation of lipid structures has proven to be a challenge task not merely due to their large number of categories but also because of the diversity of lipid isomers including varied head groups and chain lengths, unsaturation levels, substitution positions as well as double-bond locations and geometries. The vast diversity and complexity of lipid structures has stimulated the development of new characterization methods with respect to sensitivity, selectivity and specialty. Mass spectrometry (MS) is often the choice for lipid analysis providing label-free detection, high sensitivity and structural characterization capability. ${ }^{16}$ MS in combination with collision-induced dissociation (CID) is powerful in distinguishing lipid isomers bearing different head groups and chain lengths from specific fragment ions. However, the identification of the carbon-carbon doublebond positional isomers cannot be directly tackled by single stage or tandem MS. A few studies show that double-bond positional isomers may dramatically different roles in lipid physiology and pathology, 5 , ${ }^{17}$ thereby they draw extensive interest in the MS-based structural lipidomics community.
Mass spectrometric strategies for double-bond localization include (i) the use of hyphenated mass spectrometry methods such as ion mobility-mass spectrometry, ${ }^{18}$ (ii) novel gas-phase fragmentation methods such as high energy collision-induced dissociation, ${ }^{19}$ charge remote fragmentation, ${ }^{20}$ and ultraviolet photodissociation, ${ }^{21,}$ ${ }^{22}$ and (iii) derivatization methods yielding diagnostics peaks in CID. The advantages of chemical derivatization methods include easy operation and no requirements for special instrumentation, so they are potentially applicable to routine analysis. Chemical derivatization of carbon-carbon double bonds in lipids can proceed by ozonolysis, ${ }^{23-}$ ${ }^{26}$ methoxylation, ${ }^{27,28}$ methylthiolation, ${ }^{29-31}$ olefin cross-metathesis, ${ }^{32}$ Paternò-Büchi reaction, ${ }^{17,}{ }^{33-35}$ as well as epoxidation induced by plasma, ${ }^{36-39}$ and offline meta-chloroperoxybenzoic acid. ${ }^{40,41}$

Our group reported an on-demand electrochemical epoxidation method using a wire-in-a-capillary ESI emitter with a large orifice (LO-ESI emitter), which can electrochemically derivatize lipid double bonds for localization and analyze native lipids at different voltages in a single experimental run. ${ }^{42}$ This method is particularly effective for positively charged lipids (e.g. PC and PE), because electroepoxidation is initiated by positive DC voltages applied onto the ESI electrode. For negatively charged lipids, we convert the polarity by forming lithium ion adducts, functionalizing double bonds and performing the analysis in the positive ion mode.

In ESI-MS, charged droplets are conventionally produced under the excitation of a direct current (DC) high voltage. In the past two decades, two variants of ESI based on alternating current $(\mathrm{AC})^{43,44}$ and pulsed $\mathrm{DC}^{45,46}$ high voltages have been developed. In a charging-discharging cycle, the AC and pulsed DC-ESI can generate successively positively and negatively charged droplets due to the polarity change, and protonated cations and deprotonated anions can be introduced and detected by MS when switching between the positive and negative ion modes. Simultaneous production of both positive and negative ions from a single spray emitter was also observed 
in induced ESI-MS using contactless pulsed DC without changing the polarity of the applied potential. ${ }^{47,48}$ The feature of rapid polarity switching in AC and pulsed DC high voltage ESI motivates us to initiate on-demand electro-epoxidation of lipids using AC voltages.

In this work, we report an $\mathrm{AC}$-induced electrochemical mass spectrometric strategy for characterization of carbon-carbon doublebond positions in unsaturated lipids in both positive and negative ion modes. Negatively and positively charged forms of lipids are electro-epoxidized simultaneously in the positive half cycle. Negatively and positively charged lipids are detected individually in the positive and negative half cycle by MS.

\section{Methods}

Materials. Acetonitrile (ACN), water, chloroform, methanol, hydrochloric acid, oleic acid and ammonia chloride were purchased from Sigma-Aldrich (St. Louis, MO). All lipids standards were purchased from Avanti Polar Lipids (Alabaster, AL). All reagents were used without additional purification.

Mass spectrometry. An Orbitrap Velos Pro mass spectrometer (Thermo Fisher Scientific, Waltham, MA) was used for all the nanoESI-MS/MS Analysis. The following MS parameters were used for all data acquisition. Samples were ionized though the application of $1.5 \sim 3 \mathrm{kV}$ AC voltage (at peak, $100 \mathrm{~Hz}$ square wave). S-lens RF level was set to be $67.6 \%$ in the positive ion mode and $60 \%$ in the negative ion mode. Capillary temperature was set at $275^{\circ} \mathrm{C}$ in both ion modes. Full MS scans were acquired over $\mathrm{m} / \mathrm{z}$ range $200 \sim 1000$ in the positive ion mode and 100 500 in the negative ion mode. In both ion modes, the resolution was set at 60000 . Microscan number was set at 1 and maximum injection time was set at $500 \mathrm{~ms}$. Tandem mass spectra were obtained via collision-induced dissociation (CID) with a normalized collision energy of $30 \%$.

LO-ESI emitters. Emitters were pulled from single-channel borosilicate glass capillary tubes (1.5 mm O.D., 0.86 mm I.D., \#1B160-6, World Precision Instruments, Sarasota, FL) by using a P-1000 micropipette puller (Sutter Instrument Company Co., Novato, CA). Typical program for pulling the large orifice tip used in this work is: Ramp 490; Heat 525; Pull 0; Velocity 3; Time 250; Pressure 500. The average orifice of the fabricated nanoESI emitters was around $60 \mu \mathrm{m}$ (Figure S1).

LO-ESI-MS/MS Analysis. A platinum wire was inserted into the LO-ESI emitter to provide efficient electrical contact with the solution. Alternating current (AC) high voltages were applied to the electrode inside the emitter by using a homemade tunable alternating current power supply. The AC power supply consisted of a wave function generator (Model DS345, $30 \mathrm{MHz}$, Stanford Research Systems, Sunnyvale, CA), a power amplifier (Model 609D-6, 0 $\pm 4 \mathrm{kV}$, Trek, Lockport, NY) and a digital storage oscilloscope (DSO5102P, $100 \mathrm{MHz}, 1 \mathrm{GSa} / \mathrm{s}$, Hantek Electronic Co., Ltd, Qingdao, China) for the monitoring of output voltages. The LO-ESI emitter is placed 5 $\mathrm{mm}$ in front of the mass inlet.

\section{Results and Discussion}

AC induced on-demand electro-epoxidation for localizing $\mathrm{C}=\mathrm{C}$ bonds in negatively charged lipids. The LO-ESI set-up is shown in Figure 1 and the instrument parameters were the same as used in our previous work. ${ }^{42}$ The voltage is supplied by an external AC power supply instead of the DC power incorporated in the mass spectrometer. Oleic acid (FA 18:1( $\Delta 9)$ ) was selected as a proof-of-concept for demonstrating the feasibility of the AC-LO-ESI-MS strategy for negatively charged lipids.

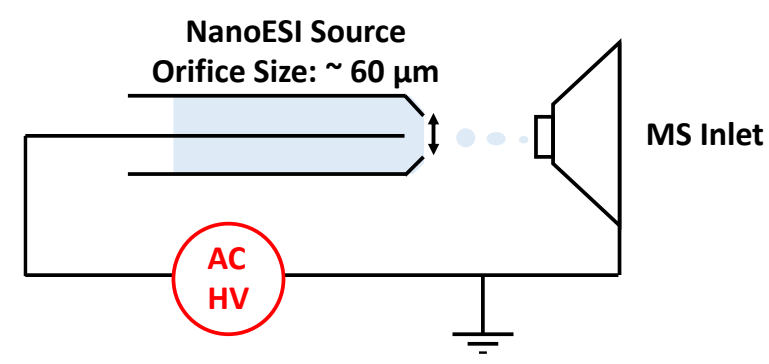

Figure 1. The configuration of AC-LO-ESI source for on demand electro-epoxidation of lipid double-bonds.

$20 \mu \mathrm{M}$ Oleic acid dissolved in acetonitrile/water $(80 / 20, \mathrm{v} / \mathrm{v})$ with $10 \mathrm{mM} \mathrm{NH}_{4} \mathrm{Cl}$ was loaded into a LO-ESI emitter with a $60 \mu \mathrm{m}$ orifice, and the mass spectrometer was operated in the negative ion mode. Upon the application of $3 \mathrm{kV}$ voltage (at peak, $100 \mathrm{~Hz}$ square wave) to the platinum wire in the emitter, a typical stable Taylor cone was formed at the tip of the emitter, and the depronated acid at $\mathrm{m} / \mathrm{z} 281.24$ was dominant in the mass spectrum (Figure 2a) without any apparent peaks corresponding to the FA epoxides, showing no electrochemical epoxidation of $\mathrm{C}=\mathrm{C}$ bonds when using the stable Taylor cone. Once the $\mathrm{AC}$ voltage was switched to $2 \mathrm{kV}$, a new peak at $\mathrm{m} / \mathrm{z} 297.24$ was detected with $65 \%$ relative abundance of the deprotonated acid at $\mathrm{m} / \mathrm{z} 281.24$ (Figure 2b), while a metastable Taylor cone could be observed. An exact 16.00 Da mass increase from $\mathrm{m} / \mathrm{z} 297.24$ above the deprotonated peak at $\mathrm{m} / \mathrm{z} 281.24$ corresponds to one oxygen atom. The results demonstrate the occurrence of electro-epoxidation of the $\mathrm{C}=\mathrm{C}$ bond in oleic acid only for the metastable Taylor cone. Upon CID of the epoxide at $\mathrm{m} / \mathrm{z} 297.24$, two diagnostic ions at $\mathrm{m} / \mathrm{z} 155$ and 171 were observed in the tandem mass spectrum (Figure $2 \mathrm{c}$ ). The diagnostic peaks have the signature $16 \mathrm{Da}$ mass difference, and their masses indicate that the doublebond position at $\Delta 9$ in oleic acid. Other fragment ions at $m / z 279.23$ and 253.25 correspond to the losses of water and carbon dioxide, respectively, showing the carboxylic substructure of oleic acid.
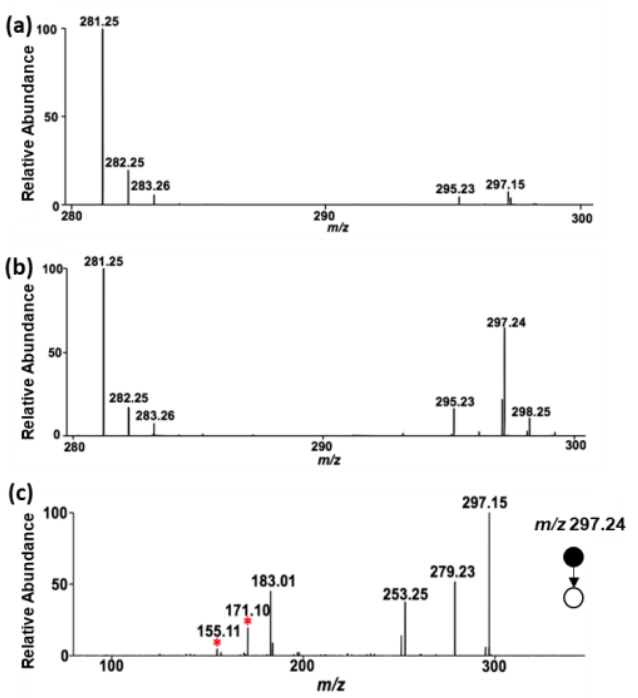
Figure 2. Full mass spectra of oleic acid (FA 18:1( $\Delta 9)$ ) at (a) $3 \mathrm{kV}$ and (b) $2 \mathrm{kV}$ (AC voltage, peak to peak, square wave, $100 \mathrm{~Hz}$ ); (c) tandem spectrum of epoxidized oleic acid, the red stars indicate the two diagnostic ions to locate doublebond positions.

We also investigated the feasibility of the AC-LO-ESI strategy in the analysis of a mixture of multiple negatively charged lipids. A mixture solution of $20 \mu \mathrm{M}$ oleic acid and $20 \mu \mathrm{M}$ vaccenic acid (FA $18: 1(\Delta 11))$ was dissolved in acetonitrile/water $(80 / 20, \mathrm{v} / \mathrm{v})$ with $10 \mathrm{mM} \mathrm{NH} 4 \mathrm{Cl}$. Upon the application of a $2 \mathrm{kV} \mathrm{AC}$ voltage, only one epoxidized peak was observed at $\mathrm{m} / \mathrm{z} 297.24$, which came from electrochemical epoxidation of oleic acid and vaccenic acid. The fragments of the epoxide at $\mathrm{m} / \mathrm{z} 297.24$ generated two groups of diagnostic ions, i.e., the ions at $\mathrm{m} / \mathrm{z} 155$ and 171 for oleic acid and the ions at $\mathrm{m} / \mathrm{z} 183$ and 199 for vaccenic acid (Figure S2). In addition, the ion intensity ratios of each group of diagnostic ions were found to be linearly correlated with the concentration ratios $(R=0.9984$, Figure S3), indicating no mutual interference and also concentration-independent epoxidation. All these results prove the success of the AC-LO-ESI-MS strategy for double-bond localization of negatively charged lipids. In addition to direct analysis of negatively charged unsaturated lipids, the AC-LO-ESI-MS strategy maintains the advantages of the DC-LO-ESI-MS strategy such as voltage-controlled chemical derivatization and low sample consumption.

$\mathrm{AC}$ induced on-demand electro-epoxidation for localizing $\mathrm{C}=\mathrm{C}$ bonds in positively charged lipids. Regardless of the successful localization of double bonds in negatively charged lipids, the half positive duration in each AC cycle is still idle for MS detection, which opens an additional window to examinepositively charged lipids. A positively charged phosphatidylcholine (PC 18:1( $\Delta 6)-18: 1(\Delta 6)$ ) was used as the model analyte to test the feasibility of the AC-LO-ESI strategy for identification of $\mathrm{C}=\mathrm{C}$ bonds in positively charged lipids. PC 18:1 $(\Delta 6)-18: 1(\Delta 6)(20 \mu \mathrm{M})$ was dissolved in acetonitrile/ water $(80 / 20, \mathrm{v} / \mathrm{v})$ containing $10 \mathrm{mM} \mathrm{HCl}$ and loaded into a LO-ESI emitter with a $60 \mu \mathrm{m}$ orifice. Upon the application of $3 \mathrm{kV}$ AC voltage, only the protonated PC peak at $\mathrm{m} / \mathrm{z} 786.67$ was observed (Figure $3 \mathrm{a}$ ) when the mass spectrometer was operated in the positive ion mode. When a low AC voltage of $2 \mathrm{kV}$ was applied instead, the mass spectrum in Figure $3 \mathrm{~b}$ demonstrates the formation of $+16 \mathrm{Da}$ and +32 Da products at $m / z 802$ and $m / z 818$, respectively. Upon CID, the tandem mass spectrum of the mono-epoxidized product at $\mathrm{m} / \mathrm{z}$ 802 clearly shows the presence of two diagnostics peaks at $m / z 618$ and 634 with a 16 Da mass shift (Figure $3 \mathrm{c}$ ), which indicates the double bond located at $\Delta 6$ position.

Furthermore, we compared electrochemical epoxidation efficiency of PC (defined as the intensity ratio of the mono-epoxide at $\mathrm{m} / \mathrm{z} 802.67$ to the intact PC at $\mathrm{m} / \mathrm{z} 786.67)$ induced by AC and DC voltages. As shown in Figure S4, the AC strategy offers slightly superior epoxidation efficiency over the DC strategy. In this case, the result demonstrates that the AC strategy offers comparable effectiveness to the DC strategy in identifying double bond positional isomers based on electrochemical epoxidation of $\mathrm{C}=\mathrm{C}$ bonds.

A mixture solution of $20 \mu \mathrm{M}$ PC $18: 1(\Delta 6)-18: 1(\Delta 6)$ and $20 \mu \mathrm{M}$ PC 18:1 $(\Delta 9)-18: 1(\Delta 9)$ in acetonitrile/water $(80 / 20, \mathrm{v} / \mathrm{v})$ containing $10 \mathrm{mM} \mathrm{NH} 4 \mathrm{Cl}$ and $1 \mathrm{mM} \mathrm{HCl}$ was also tested by the AC-LOESI strategy. Upon the application of a $2 \mathrm{kV}$ AC voltage, mono- and di-epoxidized peaks were observed at $\mathrm{m} / \mathrm{z} 802.67$ and 818.68 , respectively. The fragment of the epoxide at $\mathrm{m} / \mathrm{z} 802.67$ generated two groups of diagnostic ions, i.e., the ions at $\mathrm{m} / \mathrm{z} 618$ and 634 for PC 18:1 (6)-18:1 (6) and the ions at $m / z 660$ and 676 for PC 18:1 (9)18:1 (9) (Figure S5). In addition, a good linear relationship between the ion intensity ratios of each group of diagnostic ions and the concentration ratios $(\mathrm{R}=0.9984)$ was observed (Figure S6), indicating no mutual interference and also concentration-independent epoxidation.
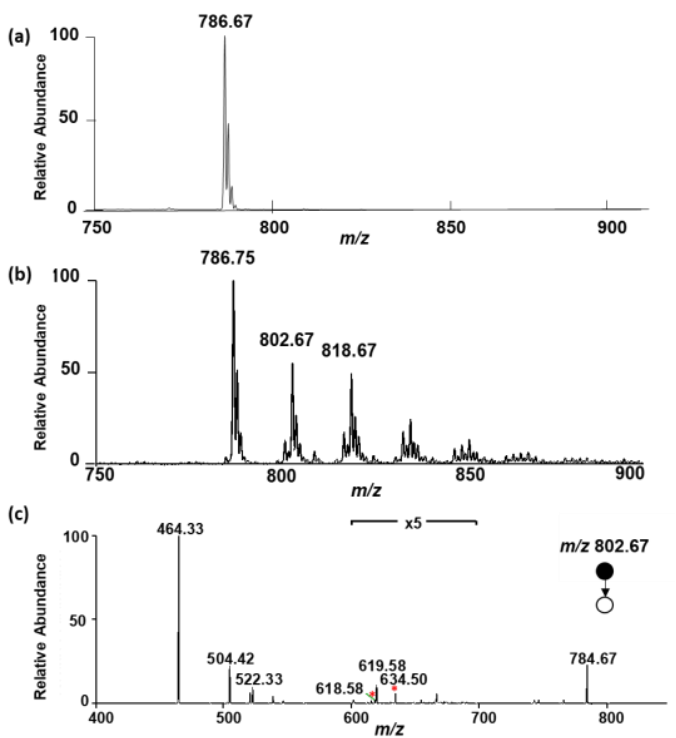

Figure 3. Full mass spectra of PC 18:1( $\Delta 6)-18: 1(\Delta 6)$ at (a) $3 \mathrm{kV}$ and (b) $2 \mathrm{kV}$ (AC voltage, peak to peak, square wave, $100 \mathrm{~Hz}$ ); (c) tandem spectrum of epoxidized PC 18:1( $\Delta 6)-18: 1(\Delta 6)$. the red stars indicate the two diagnostic ions to locate double-bond positions.

Simultaneous identification of double bonds in negatively and positively charged lipids by AC induced electro-epoxidation. The above success of the AC-LO-ESI-MS strategy for applications to lipids which ionize to give either negatively or positively charged ions urges us to further undertake the simultaneous identification of both the negatively and positively charged forms of lipids in a single run by taking full advantage of duration time in an AC cycle. The whole workflow follows in four directions (Figure 4). In the $+3 \mathrm{kV}$ and -3 $\mathrm{kV}$ half cycle, lipids of both polarities in their native forms were detected by the mass spectrometer. The protonated species in the positive ion mode and the deprotonated species in the negative ion mode were successively detected and fragmented for the characterization of head group lipid isomers. Electrochemical epoxidation of $\mathrm{C}=\mathrm{C}$ bonds in both positively and negatively charged lipids was induced in the $2 \mathrm{kV}$ half cycles. The positively charged epoxidized lipid was detected by the mass spectrometer in the same $+2 \mathrm{kV}$ half cycle, followed by the detection of the negatively charged epoxidized lipid in the next $-2 \mathrm{kV}$ half cycle. 


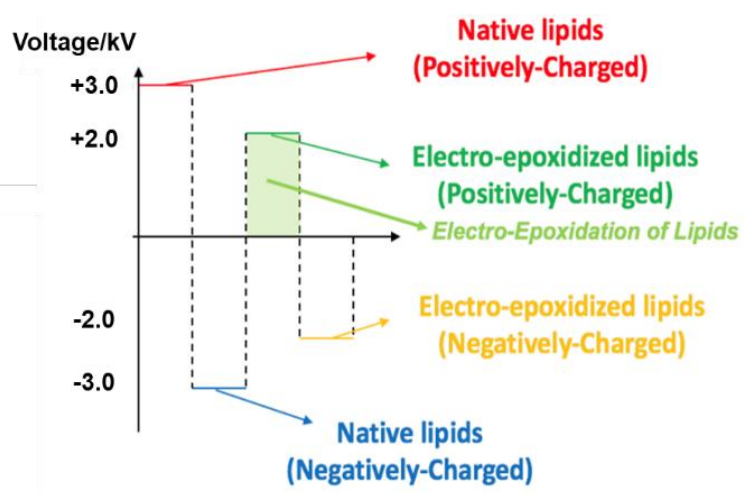

Figure 4. Lipid analysis workflow using AC-LO-ESI for the detection of positively and negatively charged forms of lipids.

Two lipids, positively charged PC 18:1( $\Delta 6)-18: 1(\Delta 6)$ and negatively charged oleic acid FA 18:1( $\Delta 9)$, were used for investigating the feasibility of simultaneous identification in one run. A mixture of $20 \mu \mathrm{M}$ PC $18: 1(\Delta 6)-18: 1(\Delta 6)$ and $20 \mu \mathrm{M}$ FA $18: 1(\Delta 9)$ was prepared in acetonitrile/water $(80 / 20, \mathrm{v} / \mathrm{v})$ containing $10 \mathrm{mM} \mathrm{NH} 4 \mathrm{Cl}$ and $1 \mathrm{mM} \mathrm{HCl}$, and then introduced to the LO-ESI emitter. When a stable electrospray was initiated at an $\mathrm{AC}$ voltage of $3 \mathrm{kV}$, the mass spectrometer detected the protonated $\mathrm{PC}$ at $\mathrm{m} / \mathrm{z} 786.67$ in the positive ion mode and the deprotonated FA at $\mathrm{m} / \mathrm{z} 281.24$ in the negative ion mode. The spectra are nearly the same with comparable intensities to the spectra of pure lipid standard analysis (Figure 2a, 3a). As the $\mathrm{AC}$ voltage was decreased to $2 \mathrm{kV}$, the mono- and di-epoxides of PC at $\mathrm{m} / \mathrm{z} 802.67$ and 818.67 , respectively, were observed in the positive ion mode (Figure S7a), and the epoxidation peak of FA with +16 Da shift at $m / z 297.24$ were thereafter detected in the negative ion mode (Figure S7b). The signal intensities of the epoxides were comparable to those in the pure lipid standards (Figure $2 b$ and $3 b$ ), demonstrating no mutual interference in the simultaneous identification. In addition, diagnostic ion pairs with a signature $16 \mathrm{Da}$ mass separation can also be detected in the tandem mass spectra using CID fragmentation (Figure S6c and S6d), confirming the positions of $\mathrm{C}=\mathrm{C}$ bonds in FA and PC. Limits of detection (LOD) for the simultaneous identification of PC 18:1( $\Delta 6)-18: 1(\Delta 6)$ and FA $18: 1(\Delta 9)$ were further evaluated. $1 \mu \mathrm{M}$ was found for both lipids (Figure S8, S9) at 3 times signal/noise level. These results demonstrate the feasibility of the AC-LO-ESI strategy in simultaneous identification of both positively and negatively charged lipids in a single trial.

When we investigated the simultaneous identification of lipids using AC-LO-ESI method, we found the chloride ions in the solvent system plays an important role in the electro-epoxidation and mass spectrometric detection. In DC-LO-ESI method, chloride ions from $10 \mathrm{mM}$ hydrochloric acid were sufficient for electrochemical epoxidation, and also beneficial to the detection of native and epoxidized $\mathrm{PC}$ ions in the protonated forms. However, excess protons suppressed the signals of the native and epoxidized FA in the deprotonated forms. A mixture of hydrochloric acid and ammonium chloride were found a good balance between the epoxidation and MS detection of lipids of both polarities. A further investigation of the ratio of the two chloride sources suggested an optimal mixture of $\mathrm{NH} 4 \mathrm{Cl}$ $(10 \mathrm{mM})$ and $\mathrm{HCl}(1 \mathrm{mM})$ for simultaneous lipid detection in both ion modes.

\section{Conclusion}

In summary, we have developed a mass spectrometric strategy of AC-induced epoxidation for double-bond localization in lipids using both positively and negatively charged unsaturated lipids simultaneously. The application of AC voltage allows simultaneous detection of lipids in both ion modes, but also the electrochemical epoxidation of both lipids in the positive half cycles. This method has the following features: i) the online electro-epoxidation allows structural characterization with no external apparatus or pre-analysis treatment of samples; ii) simultaneous detection of lipids in both ion modes simplifies the workflow for the complex sample analysis and requires a small sample consumption. The unique feature of locating double bonds in both ion modes simultaneously and the simple setup enable its wide application in many lipid-related fields.

\section{AUTHOR INFORMATION}

\section{Corresponding Author}

*E-mail: xyan@tamu.edu

\section{ACKNOWLEDGMENT}

We acknowledge support from Texas A\&M University and the Department of Chemistry.

\section{REFERENCES}

1. Quehenberger, O.; Armando, A. M.; Brown, A. H.; Milne, S. B.; Myers, D. S.; Merrill, A. H.; Bandyopadhyay, S.; Jones, K. N.; Kelly, S.; Shaner, R. L.; Sullards, C.M.; Wang, E.; Murphy, R. C.; Barkley, R. M.; Leiker, T. J.; Raetz, C.R.H.; Guan, Z.; Laird, G. M.; Six, D. A.; Russell, D. W.; McDonald, J. G.; Subramaniam, S.; Fahy, E.; Dennis, E. A., Lipidomics reveals a remarkable diversity of lipids in human plasma. J Lipid Res 2010, 51 (11), 3299-3305.

2. Thiam, A. R.; Dugail, I.J.J. o. c. s., Lipid droplet-membrane contact sitesfrom protein binding to function. 2019, 132 (12).

3. Phillips, R.; Ursell, T.; Wiggins, P.; Sens, P.J. N., Emerging roles for lipids in shaping membrane-protein function. 2009, 459 (7245), 379-385.

4. Trousil, S.; Lee, P.; Pinato, D. J.; Ellis, J. K.; Dina, R.; Aboagye, E. O.; Keun, H. C.; Sharma, R. J. C. R., Alterations of choline phospholipid metabolism in endometrial cancer are caused by choline kinase alpha overexpression and a hyperactivated deacylation pathway. 2014, 74 (23), 6867-6877.

5. Neumann, E. K.; Comi, T. J.; Rubakhin, S. S.; Sweedler, J. V. J. A. C. I. E., Lipid Heterogeneity between Astrocytes and Neurons Revealed by Single Cell MALDI - MS Combined with Immunocytochemical Classification. 2019, 58 (18), 5910-5914.

6. Yetukuri, L.; Katajamaa, M.; Medina-Gomez, G.; Seppänen-Laakso, T.; Vidal-Puig, A.; Orešič, M., Bioinformatics strategies for lipidomics analysis: characterization of obesity related hepatic steatosis. BMC Systems Biology 2007, $1(1), 12$.

7. Perry, R. J.; Samuel, V. T.; Petersen, K. F.; Shulman, G. I.J. N., The role of hepatic lipids in hepatic insulin resistance and type 2 diabetes. 2014, 510 (7503), 84-91.

8. Stegemann, C.; Pechlaner, R.; Willeit, P.; Langley, S. R.; Mangino, M.; Mayr, U.; Menni, C.; Moayyeri, A.; Santer, P.; Rungger, G. J. C., Lipidomics 
profiling and risk of cardiovascular disease in the prospective populationbased Bruneck study. 2014, 129 (18), 1821-1831.

9. Mills, G. B.; Moolenaar, W. H. J. N. R. C., The emerging role of lysophosphatidic acid in cancer. 2003, 3 (8), 582-591.

10. M Jeitner, T.; Voloshyna, I.; B Reiss, A. J. C. m. c., Oxysterol derivatives of cholesterol in neurodegenerative disorders. 2011, 18 (10), 1515-1525.

11. Bagchi, S.; Genardi, S.; Wang, C.-R. J. F. i. i., Linking CD1-restricted T cells with autoimmunity and dyslipidemia: Lipid levels matter. 2018, 9, 1616.

12. Hinterwirth, H.; Stegemann, C.; Mayr, M.J. C. C. G., Lipidomics: quest for molecular lipid biomarkers in cardiovascular disease. 2014, 7 (6), 941954.

13. Liu, Q.; Zhang, J. J. N. b., Lipid metabolism in Alzheimer's disease. 2014, $30(2), 331-345$.

14. Musso, G.; Cassader, M.; Paschetta, E.; Gambino, R. J. G., Bioactive lipid species and metabolic pathways in progression and resolution of nonalcoholic steatohepatitis. 2018, 155 (2), 282-302. e8.

15. Jha, P.; McDevitt, M. T.; Halilbasic, E.; Williams, E. G.; Quiros, P. M.; Gariani, K.; Sleiman, M. B.; Gupta, R.; Ulbrich, A.; Jochem, A. J. C. s., Genetic regulation of plasma lipid species and their association with metabolic phenotypes. 2018, 6 (6), 709-721. e6.

16. Rustam, Y. H.; Reid, G. E. J. A. c., Analytical challenges and recent advances in mass spectrometry based lipidomics. 2018, 90 (1), 374-397.

17. Ma, X.; Chong, L.; Tian, R.; Shi, R.; Hu, T. Y.; Ouyang, Z.; Xia, Y.J. P. o. t. N.A. o. S., Identification and quantitation of lipid $\mathrm{C}=\mathrm{C}$ location isomers: A shotgun lipidomics approach enabled by photochemical reaction. 2016, $113(10), 2573-2578$.

18. Kyle, J. E.; Zhang, X.; Weitz, K. K.; Monroe, M. E.; Ibrahim, Y. M.; Moore, R.J.; Cha, J.; Sun, X.; Lovelace, E. S.; Wagoner, J.J. A., Uncovering biologically significant lipid isomers with liquid chromatography, ion mobility spectrometry and mass spectrometry. 2016, 141 (5), 1649-1659.

19. Adams, J.; Gross, M. L. J. A. C., Tandem mass spectrometry for collisional activation of alkali metal-cationized fatty acids: a method for deter mining double bond location. 1987, 59 (11), 1576-1582.

20. Tomer, K. B.; Crow, F. W.; Gross, M. L. J. J. o. t. A. C. S., Location of double-bond position in unsaturated fatty acids by negative ion MS/MS. $1983,105(16), 5487-5488$

21. Williams, P. E.; Klein, D. R.; Greer, S. M.; Brodbelt, J. S. J. J. o. t. A. C. S., Pinpointing double bond and sn-positions in glycerophospholipids via hybrid $193 \mathrm{~nm}$ ultraviolet photodissociation (UVPD) mass spectrometry. 2017, 139 (44), 15681-15690.

22. Klein, D. R.; Brodbelt, J. S. J. A. c., Structural characterization of phosphatidylcholines using $193 \mathrm{~nm}$ ultraviolet photodissociation mass spectrometry. 2017,89 (3), 1516-1522.

23. Harrison, K. A.; Murphy, R. C. J. A. c., Direct mass spectrometric analysis of ozonides: Application to unsaturated glycerophosphocholine lipids. 1996, 68 (18), 3224-3230.
24. Thomas, M. C.; Mitchell, T. W.; Blanksby, S. J., Ozonolysis of Phospholipid Double Bonds during Electrospray Ionization: A New Tool for Structure Determination. Journal of the American Chemical Society 2006, 128 (1), 58-59.

25. Zhang, J. I.; Tao, W. A.; Cooks, R. G., Facile Determination of Double Bond Position in Unsaturated Fatty Acids and Esters by Low Temperature Plasma Ionization Mass Spectrometry. Analytical Chemistry 2011, 83 (12), 4738-4744.

26. Ellis, S. R.; Hughes, J. R.; Mitchell, T. W.; Blanksby, S. J. J. A., Using ambient ozone for assignment of double bond position in unsaturated lipids. 2012, 137 (5), 1100-1110.

27.Lam, C.; Jie, M. L. K. J. C.; Lipids, P. o., Fatty acids, part 5: A study of the oxymercuration-demercuration reaction of some C11-unsaturated fatty esters and methyl octadec-cis-10-en-5-ynoate. 1976, 16 (3), 181-194.

28. Minnikin, D. J. C.; Lipids, P. o., Location of double bonds and cyclopropane rings in fatty acids by mass spectrometry. 1978, 21 (4), 313-347.

29. Francis, G. W.J. C.; Lipids, P. o., Alkylthiolation for the determination of double-bond position in unsaturated fatty acid esters. 1981, 29 (4), 369-374.

30. Buser, H. R.; Arn, H.; Guerin, P.; Rauscher, S. J. A. C., Determination of double bond position in mono-unsaturated acetates by mass spectrometry of dimethyl disulfide adducts. 1983, 55 (6), 818-822.

31. Leonhardt, B.; DeVilbiss, E. J. J. o. C. A., Separation and double-bond determination on nanogram quantities of aliphatic monounsaturated alcohols, aldehydes and carboxylic acid methyl esters. 1985, 322, 484-490.

32. Kwon, Y.; Lee, S.; Oh, D. C.; Kim, S. J. A. C., Simple Determination of Double - Bond Positions in Long - Chain Olefins by Cross - Metathesis. 2011, 123 (36), 8425-8428.

33. Ma, X.; Xia, Y., Pinpointing Double Bonds in Lipids by Paternò-Büchi Reactions and Mass Spectrometry. Angewandte Chemie International Edition 2014, 53 (10), 2592-2596.

34.Ma, X.; Zhao, X.; Li, J.; Zhang, W.; Cheng, J.X.; Ouyang, Z.; Xia, Y., Photochemical Tagging for Quantitation of Unsaturated Fatty Acids by Mass Spectrometry. Analytical Chemistry 2016, 88 (18), 8931-8935.

35. Murphy, R. C.; Okuno, T.; Johnson, C. A.; Barkley, R. M. J. A. C., Determination of double bond positions in polyunsaturated fatty acids using the photochemical Paterno-Buchi reaction with acetone and tandem mass spectrometry. 2017, 89 (16), 8545-8553.

36. Cao, W.; Ma, X.; Li, Z.; Zhou, X.; Ouyang, Z., Locating Carbon-Carbon Double Bonds in Unsaturated Phospholipids by Epoxidation Reaction and Tandem Mass Spectrometry. Analytical Chemistry 2018, 90 (17), 1028610292.

37. Zhao, Y.; Zhao, H.; Zhao, X.; Jia, J.; Ma, Q.; Zhang, S.; Zhang, X.; Chiba, H.; Hui, S.-P.; Ma, X., Identification and Quantitation of $\mathrm{C}=\mathrm{C}$ Location Isomers of Unsaturated Fatty Acids by Epoxidation Reaction and Tandem Mass Spectrometry. Analytical Chemistry 2017, 89 (19), 1027010278

38. Zhao, X.; Zhao, Y.; Zhang, L.; Ma, X.; Zhang, S.; Zhang, X., Rapid Analysis of Unsaturated Fatty Acids on Paper-Based Analytical Devices via Online Epoxidation and Ambient Mass Spectrometry. Analytical Chemistry 2018, 90 (3), 2070-2078. 
39. Wan, L.; Gong, G.; Liang, H.; Huang, G., In situ analysis of unsaturated fatty acids in human serum by negative-ion paper spray mass spectrometry. Analytica Chimica Acta 2019, 1075, 120-127.

40. Feng, Y.; Chen, B.; Yu, Q.; Li, L., Identification of Double Bond Position Isomers in Unsaturated Lipids by m-CPBA Epoxidation and Mass Spectrometry Fragmentation. Analytical Chemistry 2019, 91 (3), 1791-1795.

41.Kuo, T.-H.; Chung, H.-H.; Chang, H.-Y.; Lin, C.-W.; Wang, M.-Y.; Shen, T.-L.; Hsu, C.-C., Deep Lipidomics and Molecular Imaging of Unsaturated Lipid Isomers: A Universal Strategy Initiated by mCPBA Epoxidation. Analytical Chemistry 2019, 91 (18), 11905-11915.

42. Tang, S.; Cheng, H.; Yan, X., On-Demand Electrochemical Epoxidation in Nano-Electrospray Ionization Mass Spectrometry to Locate CarbonCarbon Double Bonds. Angewandte Chemie International Edition 2020, 59 (1), 209-214.

43. Chetwani, N.; Cassou, C. A.; Go, D. B.; Chang, H.-C., High-frequency $\mathrm{AC}$ electrospray ionization source for mass spectrometry of biomolecules. Journal of the American Society for Mass Spectrometry 2010, 21 (11), 18521856.
44. Chetwani, N.; Cassou, C. A.; Go, D. B.; Chang, H.-C. J. A. c., Frequency dependence of alternating current electrospray ionization mass spectrometry. 2011, $83(8), 3017-3023$.

45.Lu, Y.; Zhou, F.; Shui, W.; Bian, L.; Guo, Y.; Yang, P. J. A. c., Pulsed electrospray for mass spectrometry. 2001, 73 (19), 4748-4753.

46. Qiao, L.; Sartor, R.; Gasilova, N.; Lu, Y.; Tobolkina, E.; Liu, B.; Girault, H. H. J. A. c., Electrostatic-spray ionization mass spectrometry. 2012, 84 (17), 7422-7430.

47. Huang, G.; Li, G.; Ducan, J.; Ouyang, Z.; Cooks, R. G. J. A. C., Synchronized inductive desorption electrospray ionization mass spectrometry. 2011, $123(11), 2551-2554$

48. Huang, G.; Li, G.; Cooks, R. G. J. A. C. I. E., Induced nanoelectrospray ionization for matrix - tolerant and high - throughput mass spectrometry. $2011,50(42), 9907-9910$ 